\title{
Thermal Modelling an LED Bulb using IR Thermography and Bayesian Deconvolution
}

\author{
https://doi.org/10.3991/ijoe.v18i03.27921 \\ Mika Maaspuro ${ }^{(\mathbb{})}$ \\ Department of Electrical Engineering and Automation, Aalto University, Espoo, Finland \\ mika.maaspuro@aalto.fi
}

\begin{abstract}
This study focuses on LED bulbs where LEDs are typically mounted on a metal core printed circuit board. A sample of LED bulbs having base E27 and a power range between 3.5-11.5 W have been measured. A thermal model will be found using thermal transient testing, infrared thermal measurements, and the Bayesian deconvolution. LED surface temperature will be measured without contact using a low-resolution IR imager. A test facility has been built which automatically switches power on and measures and records samples during the measurement cycle which typically lasts around one hour. This time duration was long enough for reaching the thermal steady-state condition. The procedure uses a measured thermal transient, first calculates the thermal impedance $Z_{\text {th }}$, then the time constant spectrum $R_{\zeta}(z)$ using the Bayesian deconvolution. An equivalent thermal network model will be found in Foster-presentation. This can be transformed to Cauer-presentation. The model is a driving point model for thermal impedance and includes thermal resistances and thermal capacitances. FEM simulation gives more detailed information about heat transfer paths and internal temperatures of an LED bulb.
\end{abstract}

Keywords - LED bulb, thermal modelling, Foster and Cauer networks, Bayesian deconvolution

\section{Introduction}

Increasing light production and electrical power of LED luminaires set new challenges for heat management in luminaires. LED bulbs have been made for replacements for incandescent bulbs having electric power up to $60 \mathrm{~W}$ or $75 \mathrm{~W}$. Typical LED replacements have power from $9 \mathrm{~W}$ to $11.5 \mathrm{~W}$. Cooling technologies can either be passive or active. Active technologies are liquid, jet impingement, electro-mechanical fan, microspray, ionic wind, piezoelectric fan [1], or thermoelectric cooling [2]. Passive cooling technologies are heat sinks, heat pipes [3], and thermal interface materials (TEM). Active cooling technologies have higher cooling capacity but they require external energy to operate. Passive cooling technologies are more reliable and cost-effective. They are also the most commonly used ones. A typical solution for an LED bulb is a heatsink which either is metal or composite design [4]. In passive cooling, the structure of a bulb and orientation of LEDs can be optimized for maximum heat removal [5]. 
For example, the so-called chimney structure [6] is an example of this kind of optimization. It is also important to arrange equal thermal conditions for all LEDs and avoid hot spots in the structure [7]. In any case, the design target is to minimize the total thermal resistance from LED chip to ambient.

There are methods to measure the thermal resistance of an LED bulb. Thermal resistance can be obtained in a steady-state condition. Electrical power is applied over a device that starts heating and finally settles all parts of the component to stable temperatures. Thermal resistance is calculated by dividing the temperature difference by the applied electrical power. Thermal resistance calculated by this way is a measure for the hole component. This method does not give an idea what are the internal thermal resistances and capacitances [8]. To fully understand thermal conditions inside a component a time-dependent model needs to be developed. Some methods can provide this information. In the following paragraph, the Bayesian deconvolution method is shortly presented.

The target is to create a thermal model for an LED bulb that can be used to calculate LED surface or junction temperature as a function of time after a power-on transient. The model should include thermal impedances equivalent to time constants related to the physical structure and material characteristics of the LED bulb. Such a thermal model has an analogy to an electrical circuit. The analog is the following: Thermal resistance is equivalent to electrical resistance, heat capacitance to electrical capacitance, heat flow to electrical current, and temperature to electrical voltage. Therefore, the model can be imported into an electric circuit simulator. Time-dependent simulation execution in a simulator results in node voltages that are equivalents to temperatures in the given locations.

\section{Method-solving thermal resistance using deconvolution}

In the transient thermal method, a step like input power is initiated at time zero and the increasing temperature of LEDs are measured continuously. In this application, the surface temperature is measured over a relatively long period of time. In the beginning, the temperature is sampled as fast as possible to detect the fast time constants. Later the sampling speed will be lowered to avoid collecting a waste amount of data. It is beneficial to change to a logarithmic time base. In this study, it was chosen to record a constant number of samples per decade.

The requirements for using the method are: heat path is one-dimensional and heat conducts from part to part through adiabatic surfaces and finally ends to an ideal heatsink. This is hardly ever the case as in normal cases heat spreads both horizontally and vertically in the structure through several concurrent paths. However, the method has been used in cases heat could have several paths to proceed. The other requirement is that thermal resistances and capacitances are constants within the temperature range. This means that temperature change is minor or moderate. [9] suggests that this condition exists if $\Delta \mathrm{T}_{\max }<50^{\circ} \mathrm{C}$. Principally not even the thermal conductivity is a constant in step like heat flow transitions [10].

The basic equation covering one-dimensional heat flow and showing the relationship between temperature change, thermal resistance, and thermal capacitance is: 


$$
P=d Q / d t=\left(T_{1}-T_{0}\right) / R_{t h}=C_{t h} d T / d t
$$

Integrating the equation above leads to the solution for $T(t)$ in case there is a single time constant.

$$
T(t)=T_{1}+\left(T_{0}-T_{1}\right) e^{-t /\left(R_{t h} C_{t h}\right)}=R_{t h} \cdot \Delta P \cdot e^{-t /\left(R_{t h} C_{t h}\right)}
$$

The thermal impedance including the effect of thermal capacitance in case of power step input is:

$$
Z_{t h}(t)=R_{t h}\left(1-e^{-t / \tau}\right)
$$

The measured heating curve has a form as shown in Figure 3. in logarithmic time base. There are several time constants which forms the curve. For the case of multiple time constants $Z_{t h}(t)$ is:

$$
\begin{gathered}
Z_{t h}(t)=\sum_{i=1}^{n} R_{t h_{i}} \cdot\left(1-e^{-t / \tau_{i}}\right) \\
\tau_{i}=R_{t h_{i}} \cdot C_{t h_{i}}
\end{gathered}
$$

The discrete time constant spectrum is a presentation where each discrete peak represents specific $R_{t h}$ at a specific time. Total $R_{t h}$ is the sum of each $R_{t h}$.

The spectrum can be changed to a continuous time constant spectrum (as Figure 7). In that case, the total thermal impedance is an integral equation.

$$
\begin{gathered}
Z_{t h}(t)=\int_{-\infty}^{\infty} R_{\zeta}(\zeta)\left(1-e^{-t / e^{\zeta}}\right) d \zeta \\
\zeta=\ln (\tau)
\end{gathered}
$$

It is a common practice to name $Z_{t h}(t)=a$. Calculating derivatives on both sides results in Equ. (8)

$$
\frac{d}{d z} a(z)=\int_{-\infty}^{\infty} R_{\zeta}(\zeta)\left(e^{z-\zeta-e^{2-\zeta}}\right) d \zeta
$$

Defining $z$, and choosing $w_{z}(z)$

$$
\begin{gathered}
z=\ln (t) \\
w_{z}(z)=e^{z-e^{z}} \\
\frac{d}{d z} a(z)=\int_{-\infty}^{\infty} R_{\zeta}(\zeta) w_{z}(z-\zeta) d \zeta
\end{gathered}
$$


This presents a convolution between the spectrum of thermal resistance and the weighting function.

$$
\frac{d}{d z} a(z)=R_{\zeta}(z) \otimes w_{z}(z)
$$

$R_{\zeta}(z)$ can be solved using deconvolution.

$$
R_{\zeta}(z)=\left[\frac{d}{d z} a(z)\right] \otimes^{-1} w_{z}(z)
$$

The deconvolution can be solved using iterative Richardson-Lucy algorithm. Typically, at least 1000 iterations will be needed [11]. The algorithm is available as a function in Matlab imaging processing toolbox.

$$
R_{n+1}(z)=R_{n}(z) \cdot\left(\frac{d a(z) / d z}{w_{z}(z) \otimes R_{n}(z)}\right) \oplus w_{z}(z)
$$

$R_{n+1}(z)$ is the n:th iteration result for $R_{t h}, a^{\prime}(z)$ is the gradient of temperature, $w_{z}(z)$ is the weighting function. $\otimes$ is the convolution operator, $\oplus$ is the correlation operator.

During the iterations the error reduces. It is also possible that after some number of iterations the error starts to increase. To end the iterations at the best moment, the error can be calculated at each iteration step.

$$
\Delta R=\frac{\Sigma_{z}\left(R_{n+1}(z)-R_{n}(z)\right)}{\Sigma_{z} R_{n}(z)}
$$

Thermal resistances are solved by integrating

$$
R_{t h, i}=\int_{z_{i}-\Delta}^{z_{i}+\Delta} R(\zeta) d \zeta
$$

The total thermal resistance would be

$$
R_{t h}=\int_{-\infty}^{\infty} R(\zeta) d \zeta
$$

The algorithm is noise-sensitive. It is necessary to somehow filter $a^{\prime}(z)$. Moving average filtering is often used, although other methods exist. Noise is also enhanced by deconvolution. Generally, the accuracy is related to the half-width of $w_{z}(z)$ [9].

The algorithm results in thermal resistances of Foster network. The thermal capacitance can then be calculated using the time constant value. Using a transformation 
widely used with electrical circuits, the Foster network can be transformed to Cauer network. The result is a driving point presentation for $Z_{t h}$ and the components of Cauer network can be directly related to thermal resistances and capacitances of the structure and material parameters.

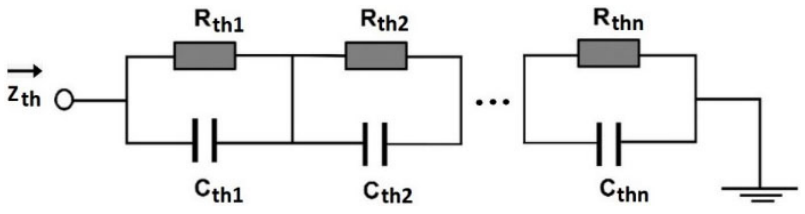

Fig. 1. Foster network presentation for a thermal impedance

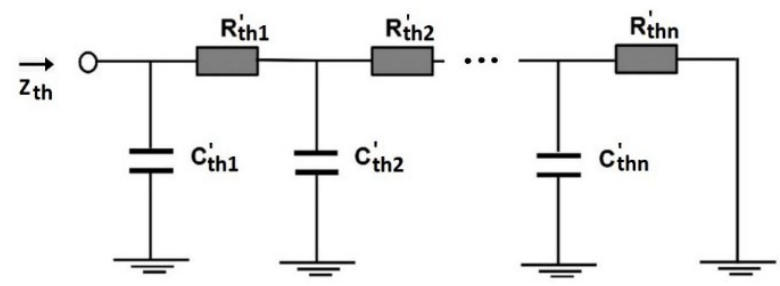

Fig. 2. Cauer network presentation for a thermal impedance

[12] presents an improved algorithm to reduce noise superimposed in the measurement signal. In their algorithm measured signal is first Fourier transformed (weighted DFT) and then filtered in a logarithmic frequency domain. Then an inverse DFT restores the signal in a time domain. Finally, Bayesian deconvolution is used to obtain the time constant spectrum. This algorithm does not use the moving average filtering which cannot fully eliminate noise which will be emphasized in calculating $d a(z) / d z$.

\section{$3 \quad$ Measurement device}

Figure 3 shows the schematic presentation of the developed measurement device. The device is based on a low-resolution IR-imager measuring through $7 \mathrm{~mm}$ hole in the LED bulb. The imager has a resolution of $32 \times 24$ pixels and a viewing angle of $55^{\circ} \times 35^{\circ}$. The resolution is enhanced using interpolation. IR-imager features relatively fast temperature measurements although the measurement accuracy is modest. Accuracy is affected on many parameters like frame sampling rate, calibration, surface emissivity, etc. The frame sampling rate can be set low, in this application around 4 frames per second, to reduce noise and reach maximum accuracy. The IR-imager is on a module including STM32 microcontroller. The imager communicates via $\mathrm{I}^{2} \mathrm{C}$ bus with the controller. The controller provides UART-bus and a limited but an easier to use interface to be operated externally. Python software is developed for PC. The software reads data from the IR-imager, set time base for the measurements, initiates and ends the measurement cycle, switch on and off LED-bulb power and finally write 
data records into a file. Controlling the LED bulb power is based on an opto-isolated electro-mechanical switch. The switching time of such devices is around 5-15 ms. This is still less than a power-on time of LED-bulb drivers, estimated to be $<200 \mathrm{~ms}$. Due to the switching delays time constants below 1 second cannot be resolved with this measurement device. Attention has been paid to the longer time constants.

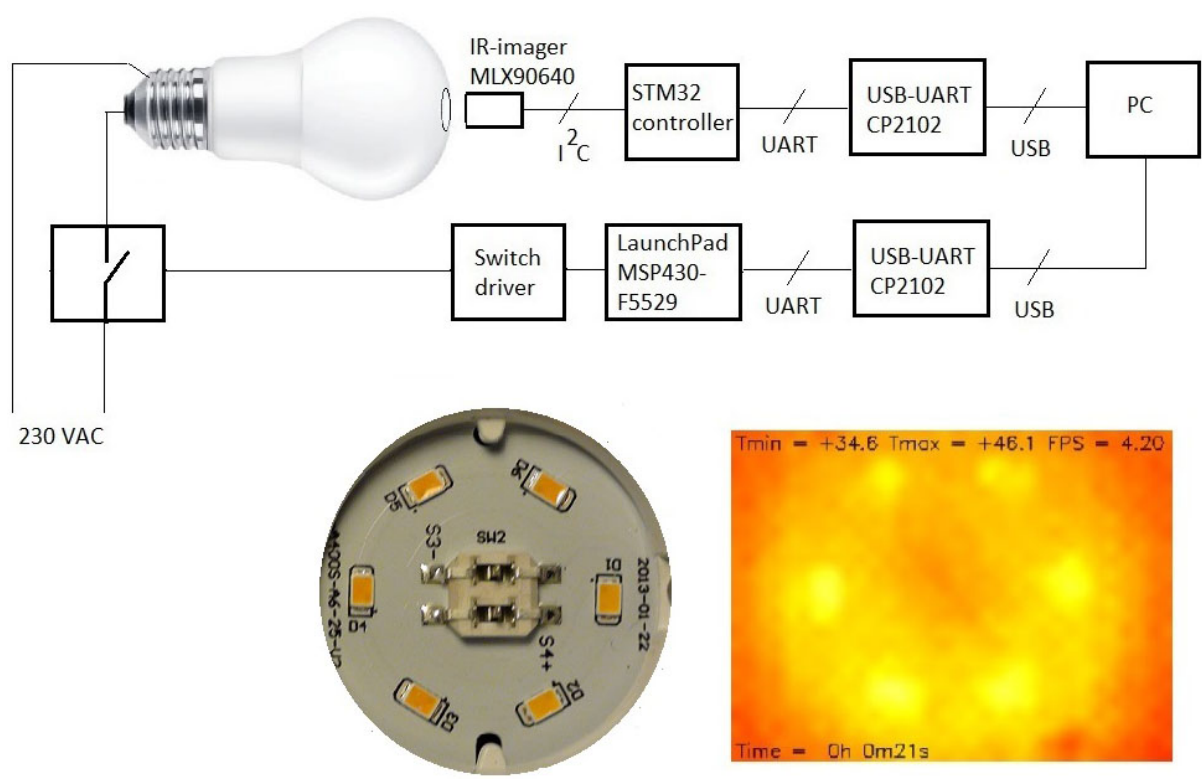

Fig. 3. The measurement device based on low-resolution IR-imaging. Image of a MPCB where there are 6 LEDs visible. The maximum frame rate (FPS) is around 4 images/s

\section{$4 \quad$ Measured LED bulbs}

All measured LED bulbs have a colour temperature of $2700 \mathrm{~K}$. Their power range is from $3.5 \mathrm{~W}$ to $11.5 \mathrm{~W}$. Bulbs $1-5$ have dome sizes of $60 \mathrm{~mm}$ and bulb 6 of $77 \mathrm{~mm}$. Bulb 3 has a bit heavier body with a thicker aluminium heatsink. Bulbs 1-2 have a full plastic body. LEDs are off course mounted on a metal substrate printed circuit board (MCPCB) and the base is round shaped steel. Bulbs 4-6 have a composite body, a thin aluminium layer $(0.3 \mathrm{~mm})$ enclosed with plastic.

Table 1 lists the bulbs with electrical powers, estimated heat powers of LEDs, and the measured LED surface temperatures. Figure 4 shows the heating curves of the bulbs. Figure 5 shows the weighting function $\mathrm{w}_{\mathrm{z}}(\mathrm{z})$ used in the deconvolution. Figure 6 shows the smoothed $\mathrm{da}(\mathrm{z}) / \mathrm{dz}$ for bulb no: 4 . Figure 7 shows the continuous time constant spectrum for bulb no: 4 . In Figure 8 the thermal model for bulb no: 4 is compared with the measured values. They match quite well and the steady-state value matches exactly. Using least-squared optimization for the model adjusting only capacitive values results in almost identical curves. Tables 2-5 list the component values of the models. 
Table 1. Electrical powers of the LED bulbs and the measured LED surface temperatures. Ambient $25^{\circ} \mathrm{C}$

\begin{tabular}{|c|c|c|c|}
\hline LED Bulb & $\mathbf{P}[\mathbf{W}]$ & $\mathbf{P}_{\mathbf{h}, \mathbf{L E D}}[\mathbf{W}]$ & $\mathbf{T}\left[{ }^{\circ} \mathbf{C}\right]$ \\
\hline 1 & 3.5 & 2.38 & 75 \\
\hline 2 & 4.5 & 3.06 & 85 \\
\hline 3 & 6.3 & 4.28 & 77 \\
\hline 4 & 9.0 & 6.12 & 130 \\
\hline 5 & 9.4 & 6.39 & 114 \\
\hline 6 & 11.5 & 7.82 & 88 \\
\hline
\end{tabular}

The main heat paths in an LED bulb are: from the LEDs to MCPCB, from MCPCB via the heatsink to ambient, from MCPCB via the bulb to ambient, from MCPCB via the heatsink and the base to ambient. As only the first path is in series and the others are in parallel, the elements of Cauer network cannot be matched to certain parts of the geometry. The sums of resolved thermal resistances match well for all the bulbs.

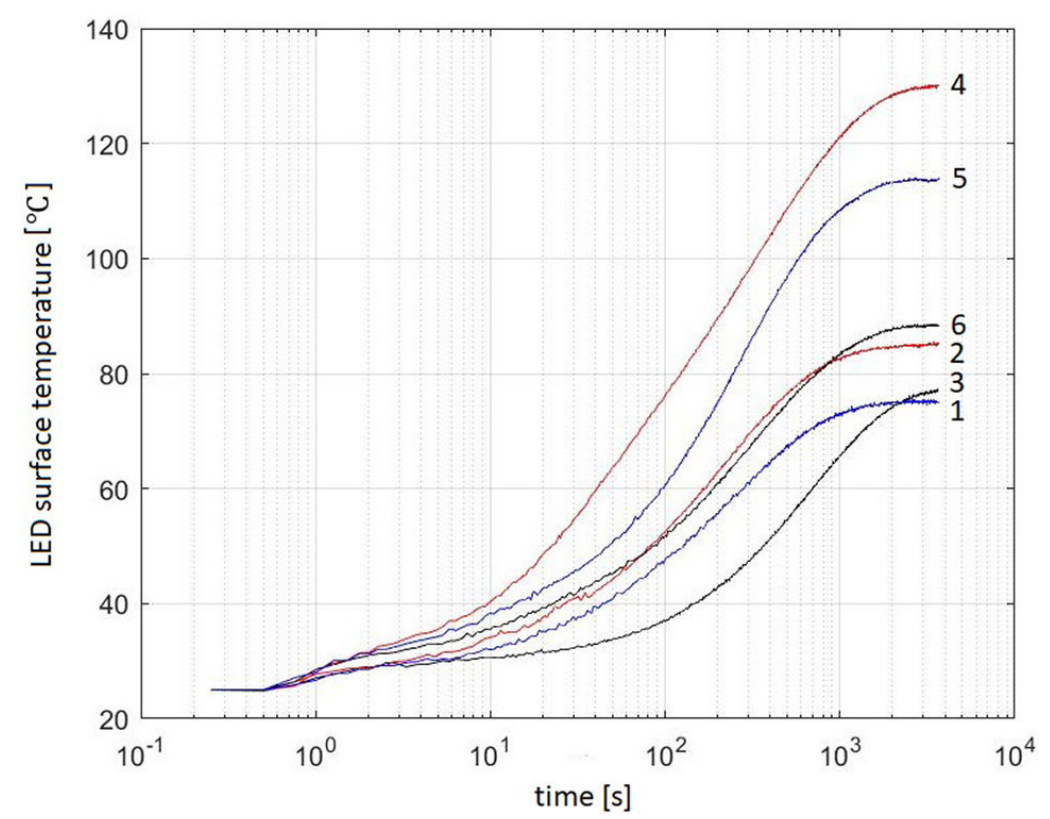

Fig. 4. The measured LED surface temperatures. LED bulbs no:1-6 


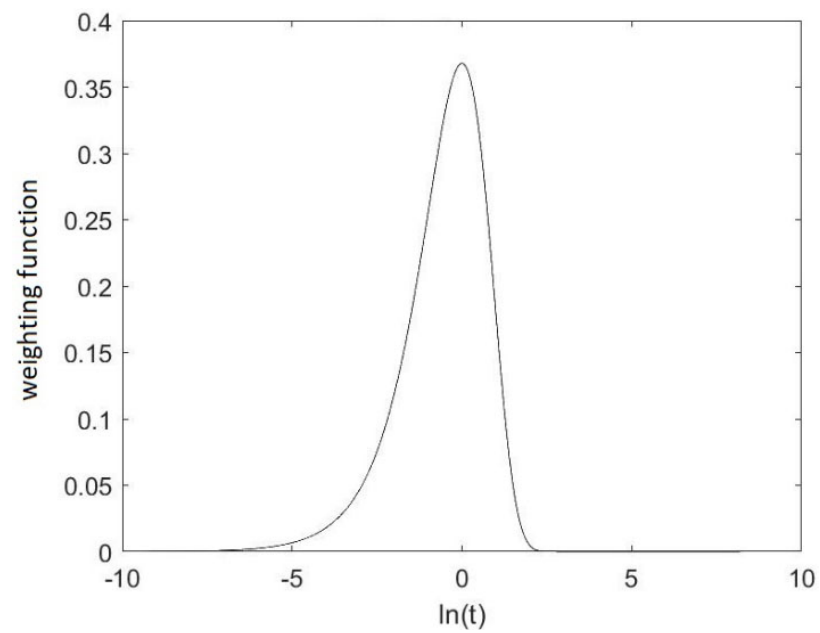

Fig. 5. The weighting function $w_{z}(z)$ used in the deconvolution

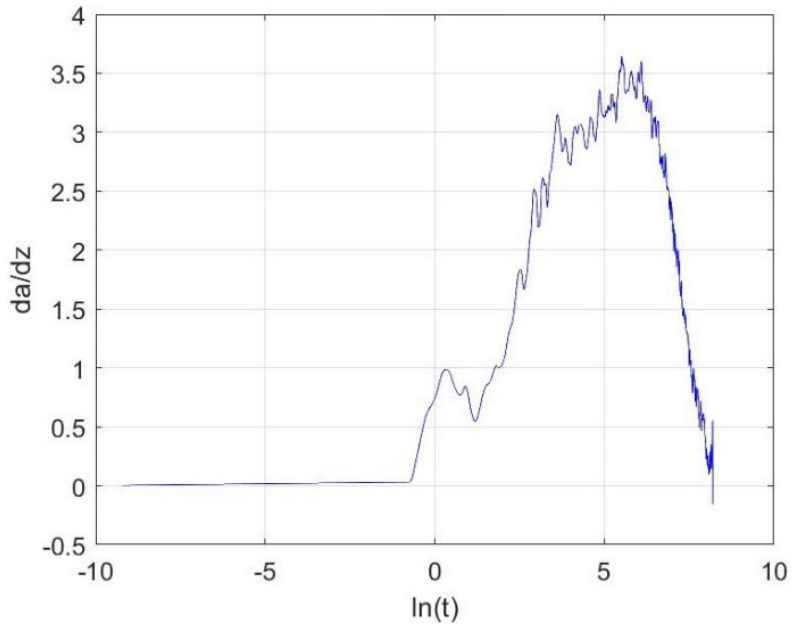

Fig. 6. The measured $d a / d z$ of LED bulb no:4 


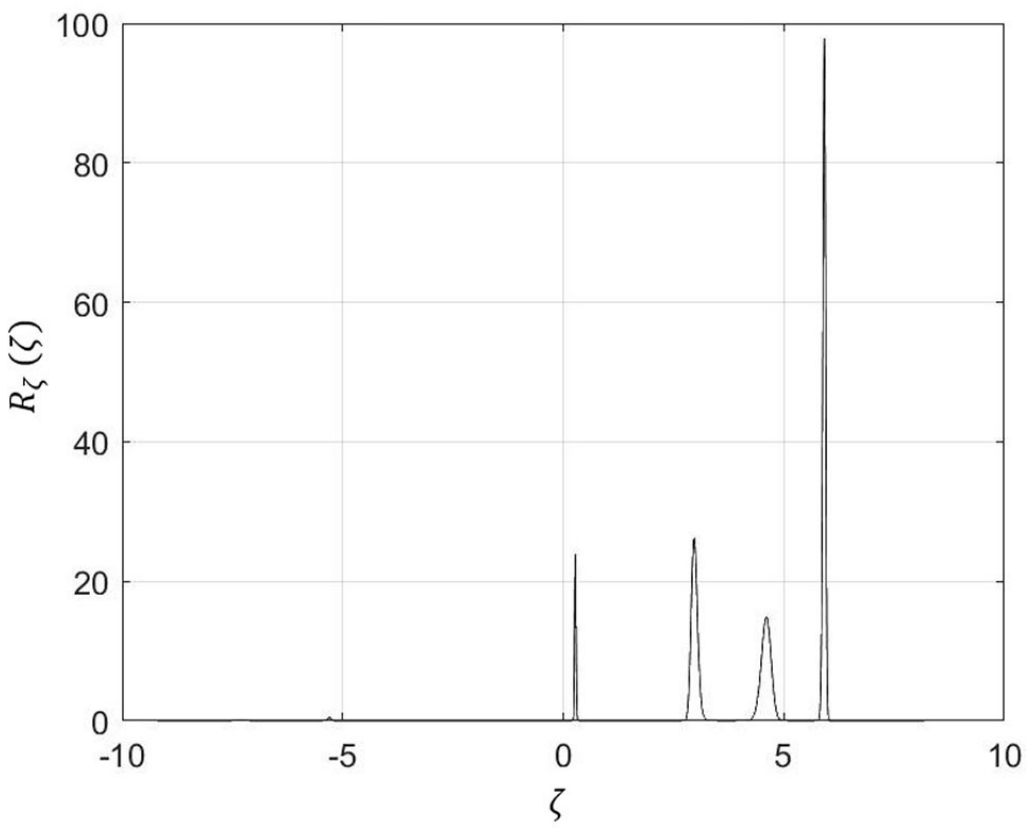

Fig. 7. Time constant spectrum of LED bulb no: 4. Richardson-Lucy algorithm, 2700 iterations

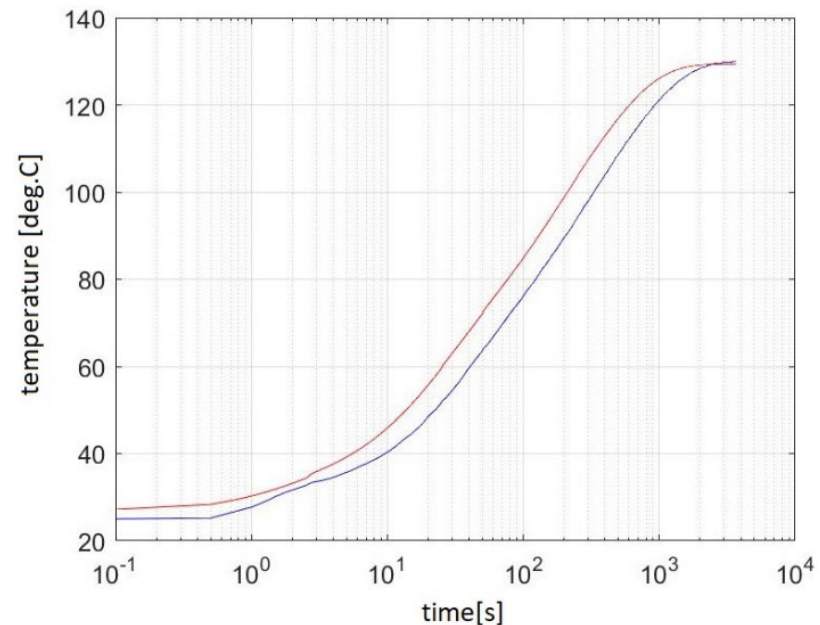

Fig. 8. Surface temperature of LED bulb no:4 calculated using model based on results of deconvolution (red) and the measured temperature (blue) 


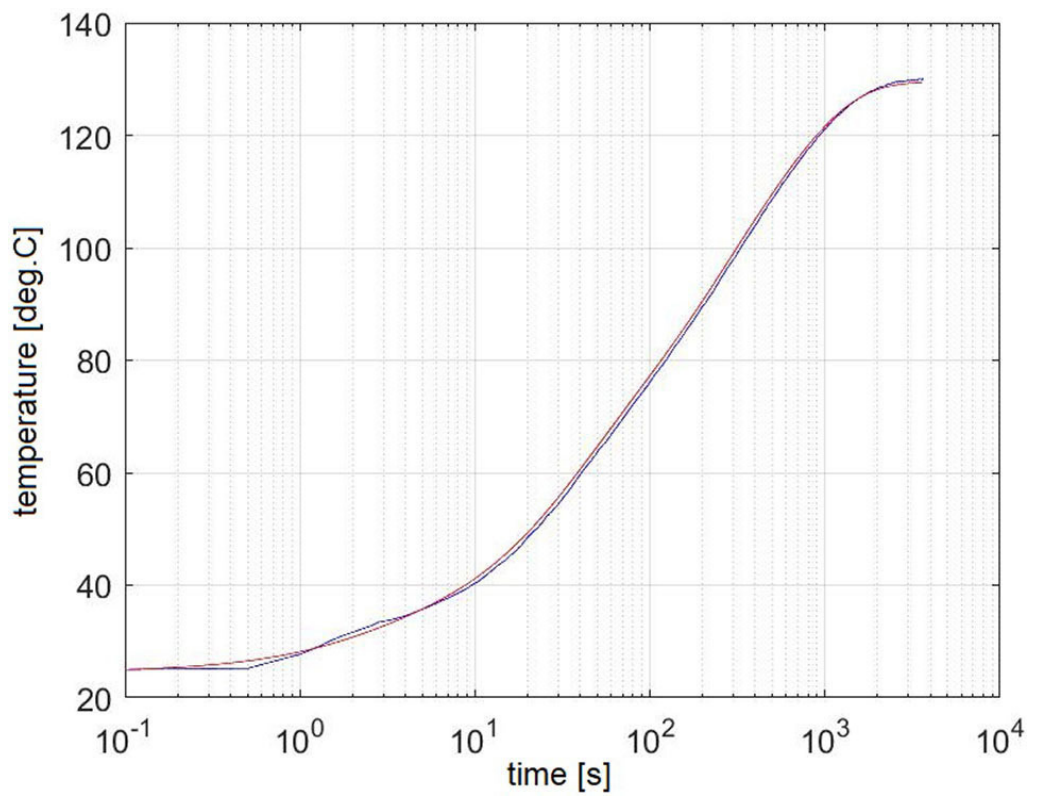

Fig. 9. The least-squares optimized solution for LED bulb no:4 (red) and the measured temperature (blue)

Table 2. Thermal resistances in Foster network

\begin{tabular}{|c|c|c|c|c|c|c|}
\hline Bulb & $\mathbf{R 1}$ & $\mathbf{R 2}$ & $\mathbf{R 3}$ & $\mathbf{R} 4$ & $\mathbf{R 5}$ & $\mathbf{R}_{\text {th }}$ \\
\hline $\mathbf{1}$ & 1.54 & 1.19 & 2.91 & 14.37 & 1.92 & 21.93 \\
\hline $\mathbf{2}$ & 0.90 & 1.86 & 4.53 & 11.58 & 0.82 & 19.69 \\
\hline $\mathbf{3}$ & 1.01 & 0.10 & 0.50 & 10.54 & & 12.15 \\
\hline $\mathbf{4}$ & 0.95 & 4.58 & 4.09 & 7.52 & & 17.14 \\
\hline $\mathbf{5}$ & 0.87 & 1.09 & 2.86 & 7.82 & 1.39 & 14.02 \\
\hline $\mathbf{6}$ & 0.01 & 0.54 & 2.06 & 5.50 & & 8.12 \\
\hline
\end{tabular}

Table 3. Heat capacitances in Foster network

\begin{tabular}{|c|c|c|c|c|c|}
\hline Bulb & C1 & C2 & C3 & C4 & C5 \\
\hline $\mathbf{1}$ & 1.10 & 21.56 & 16.83 & 17.76 & 540.74 \\
\hline $\mathbf{2}$ & 1.86 & 5.55 & 20.31 & 24.02 & 1386.30 \\
\hline $\mathbf{3}$ & 1.57 & 108.86 & 199.71 & 61.75 & \\
\hline $\mathbf{4}$ & 2.49 & 6.59 & 36.96 & 75.66 & \\
\hline $\mathbf{5}$ & 2.53 & 10.44 & 47.10 & 43.88 & 730.84 \\
\hline $\mathbf{6}$ & 2.12 & 13.31 & 61.94 & 82.75 & \\
\hline
\end{tabular}


Table 4. Thermal resistances in Cauer network

\begin{tabular}{|c|c|c|c|c|c|c|}
\hline Bulb & $\mathbf{R 1}$ & $\mathbf{R 2}$ & $\mathbf{R 3}$ & $\mathbf{R} 4$ & $\mathbf{R 5}$ & $\mathbf{R}_{\text {th }}$ \\
\hline $\mathbf{1}$ & 2.14 & 7.97 & 6.29 & 4.61 & 0.93 & 21.93 \\
\hline $\mathbf{2}$ & 1.93 & 3.45 & 10.16 & 3.78 & 0.38 & 19.69 \\
\hline $\mathbf{3}$ & 1.11 & 1.10 & 5.47 & 4.48 & & 12.15 \\
\hline $\mathbf{4}$ & 2.01 & 6.15 & 5.93 & 3.04 & & 17.14 \\
\hline $\mathbf{5}$ & 1.52 & 1.91 & 7.92 & 2.24 & 0.43 & 14.02 \\
\hline $\mathbf{6}$ & 0.01 & 1.01 & 4.85 & 2.24 & & 8.12 \\
\hline
\end{tabular}

Table 5. Heat capacitances in Cauer network

\begin{tabular}{|c|c|c|c|c|c|}
\hline Bulb & C1 & C2 & C3 & C4 & C5 \\
\hline $\mathbf{1}$ & 0.94 & 5.60 & 12.34 & 17.89 & 1071.40 \\
\hline $\mathbf{2}$ & 1.23 & 3.42 & 8.52 & 49.62 & 2893.60 \\
\hline $\mathbf{3}$ & 1.49 & 34.66 & 15.48 & 46.58 & \\
\hline $\mathbf{4}$ & 1.68 & 4.27 & 29.30 & 130.15 & \\
\hline $\mathbf{5}$ & 1.87 & 7.56 & 15.88 & 108.64 & 2173.80 \\
\hline $\mathbf{6}$ & 1.74 & 7.97 & 28.66 & 137.34 & \\
\hline
\end{tabular}

\section{Simulation case}

The LED surface measurement cannot solve heat paths in an LED bulb. FEM model simulations can solve detailed information about the heat transferring in the structure. The designed FEM model matches the geometries and materials of bulb 3 . The model has two main parts: The bulb itself and a simple luminaire outside the bulb. Figure 10 shows the geometry. Its main parts are the base, the heatsink, the metal core printed circuit board MCPCB, the driver board, and the bulb. In some LED bulbs there is no separate driver board and all components are mounted on the MCPCB. In this model like in bulb 3, the heatsink has a form of a cone and thickness of 3-4 mm. The bulb has a diameter of $60 \mathrm{~mm}$. Figure 11 shows the bulb and the luminaire.

The model utilizes one plane symmetry (without the driver board) and therefore it is possible to include only half of the bulb and a luminaire is included in the model. The mesh has around 700000 elements which still can be easily simulated using the available computing cluster. The model allows setting the thickness of the aluminium heatsink between 1-3 mm. The time-dependent simulation from the initial power step to the steady-state condition is around one hour. In the simulations, the bulb faces downwards while natural convection conditions prevail. The temperature of the base sets to $38^{\circ} \mathrm{C}$ according to the measured temperatures of the luminaire. Ambient, in that case, is $20^{\circ} \mathrm{C}$. In the measurements, the ambient was $25^{\circ} \mathrm{C}$, and this should be kept in mind when comparing the measured and simulated values. 


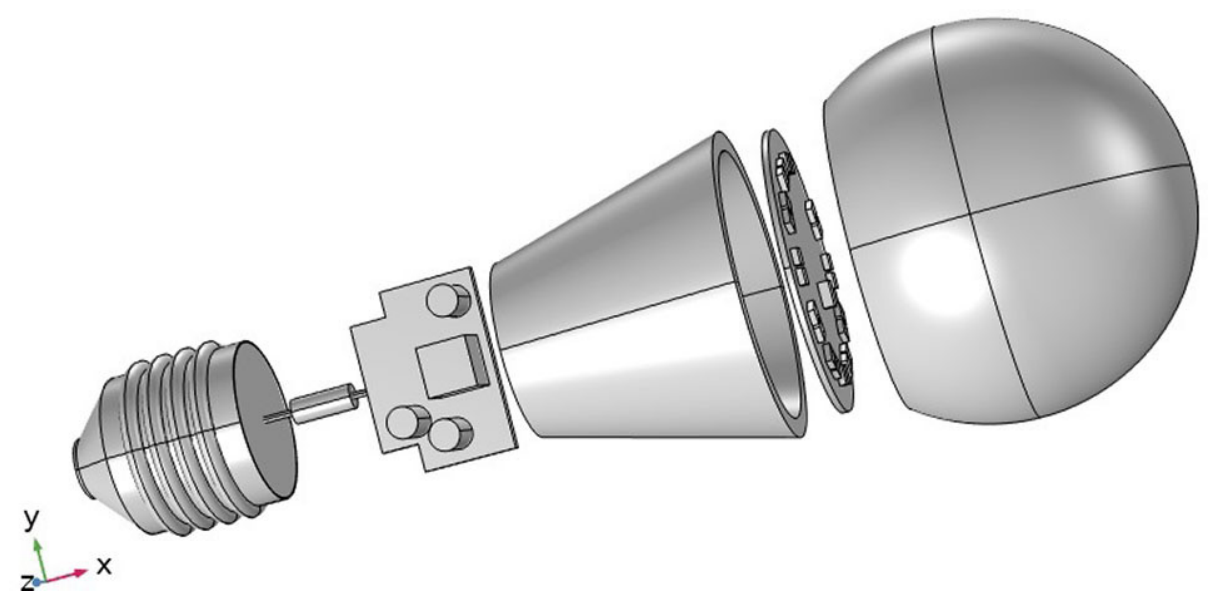

Fig. 10. FEM-simulation model for an LED-bulb (exploded view). Parts: a bulb, MCPCB, a heatsink, a driver board and a base
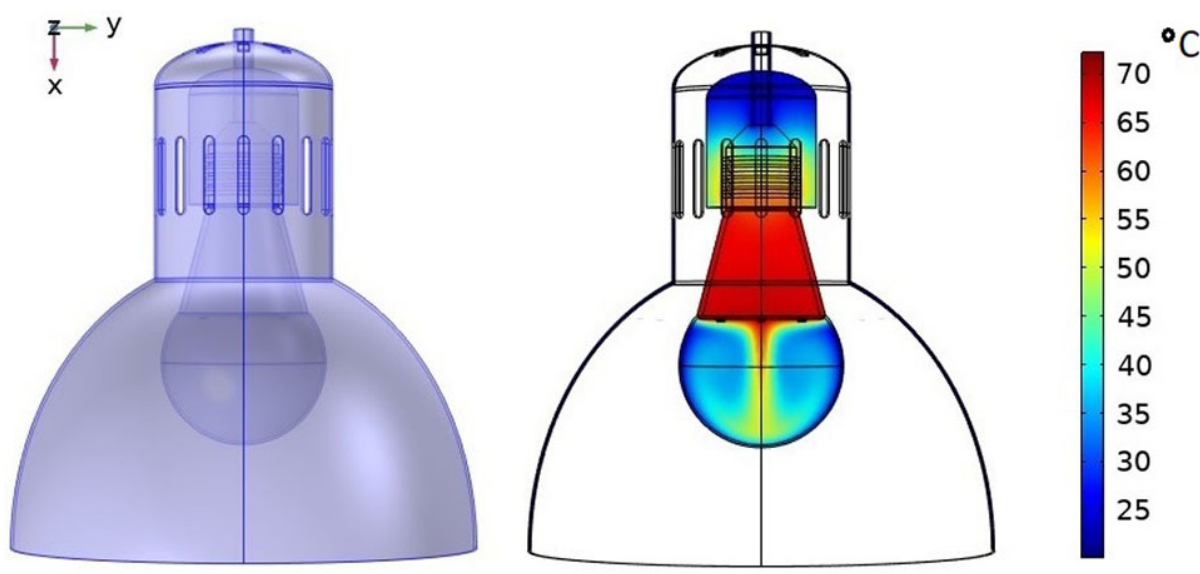

Fig. 11. Simulated LED bulb with a luminaire and the thermal solution at $\mathrm{t}=1 \mathrm{~h}$ (slice view)

\subsection{Simulation results}

The simulated heating curve is presented in Figure 12. This can be compared to the curve of bulb 3 in Figure 4. The thermal steady-state situation will be reach around one hour. LED packages and the heatsink set around $71^{\circ} \mathrm{C}$ when the ambient is $20^{\circ} \mathrm{C}$. Figure 13 shows heat paths through the base, the heatsink, and the bulb. During approximately the first 4 minutes, heat is conducted equally via the base and the heatsink. Later the flow via the heatsink dominates. In steady-state conditions, heat conducts mainly via the heatsink (56\%), and the base (31\%), and the bulb (13\%). These results are for a heatsink with a thickness of $3 \mathrm{~mm}$. 


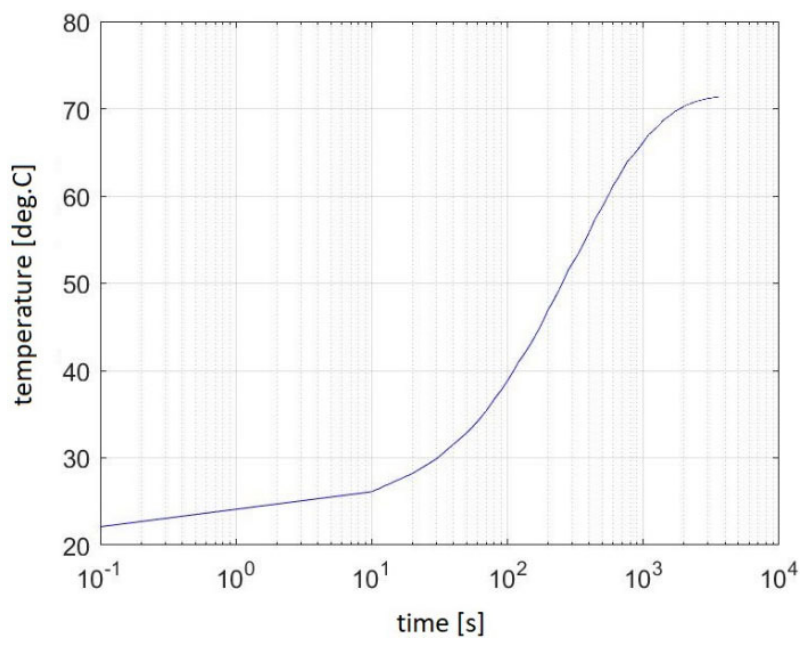

Fig. 12. Simulated LED surface temperature for the LED bulb resembling bulb no: 3 . Ambient $20^{\circ} \mathrm{C}$

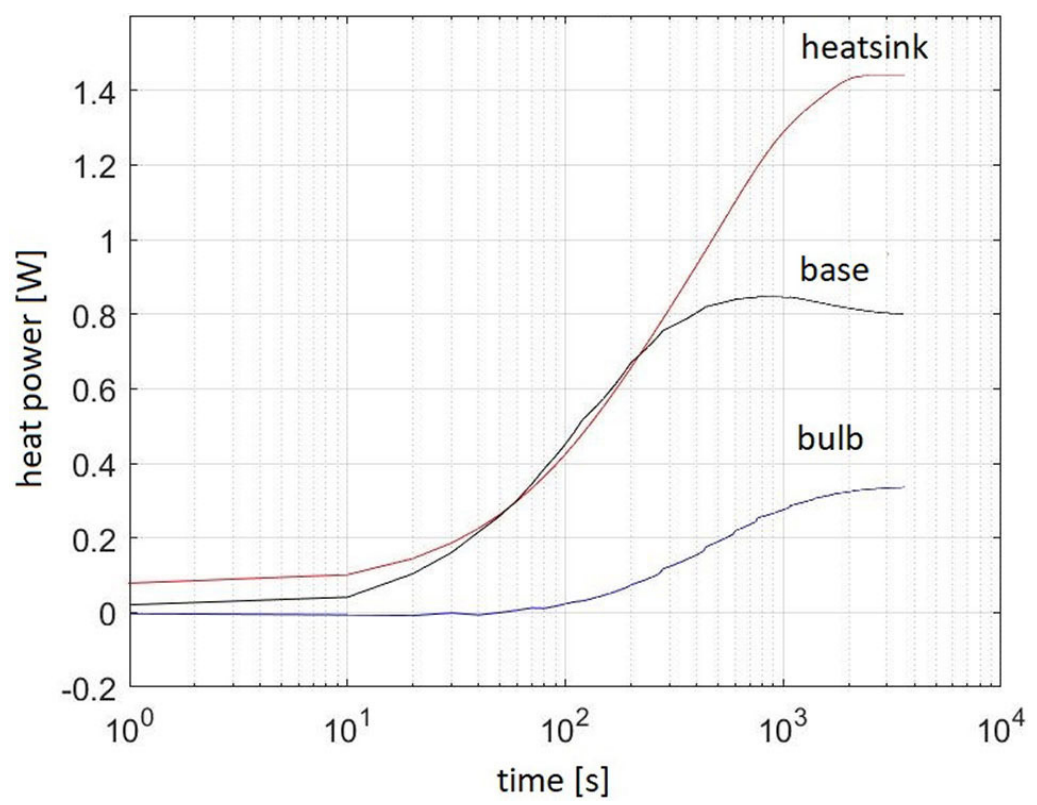

Fig. 13. Simulated heat flows in the LED bulb resembling bulb no: 3 . Ambient $20^{\circ} \mathrm{C}$ 


\section{Conclusions}

LED bulbs with electrical power consumption from $3.5 \mathrm{~W}$ to $11.5 \mathrm{~W}$ were measured. Some simplifications were made. It can be assumed that the efficiency of an LED driver is around $85 \%$ and $20 \%$ of the LED power transferred to light and the rest to heat. LED driver parts can be mounted on the same MCPCB as the LEDs or be located on a separate board. The major problem and the limitation of this study is that the driver also generates some heat. Should this heat be ignored or added to the LED power? In case the driver is on the same board and the heat is added to the LED power the calculated thermal resistance from the board to ambient is closer to reality. Using the Bayesian deconvolution method, the time constant spectrum for an LED bulb was calculated. From the spectrum, Foster-presentation for the thermal network was defined and using an algorithm converted to an equivalent Cauer-presentation. The solution was focused on using the least-squares optimization method. The model predicts LED surface temperature vs. time for the given LED heating power. Measurements show that there are large differences between the LED surface and hence the LED junction temperatures. During the past years, LED bulb heatsinks have become lighter with less metal inside and therefore the LEDs operate at a higher temperature. Among the studied LED bulbs there was no metal heatsink in those bulbs having electrical power under $5 \mathrm{~W}$. Ignoring the metal heatsink reduces manufacturing costs but also the lifetime of the bulbs. Measured LED surface steady-state temperatures range from $75^{\circ} \mathrm{C}$ to $130^{\circ} \mathrm{C}$ (ambient $25^{\circ} \mathrm{C}$ ). FEM simulations show that in an LED bulb approximately $56 \%$ of the heat conducts through the heatsink, $31 \%$ through the base, and $13 \%$ through the bulb. This result is for a simple luminaire and a case where the bulb is heading downwards in steady-state and external natural convection conditions.

\section{$7 \quad$ References}

[1] M. Maaspuro, "Novel Ideas for Thermal Management of Filament LED Light Bulbs", iJOE International Journal of Online and Biomedical Engineering, pp. 60-73, Vol. 17, No. 8, 2021. https://doi.org/10.3991/ijoe.v17i08.23695

[2] D. Zhong, H. Qin, C. Wang, Z. Xiao, "Thermal Performance of Heatsink and Thermoelectric Cooler Packaging Designs in LED”, 11 th. International Conference on Electronic Packaging Technology \& High Density Packaging, pp. 1377-1381, IEEE, 2010. https://doi. org/10.1109/ICEPT.2010.5582819

[3] Yu.E. Nikolaenko, D.V. Pekur, V.M. Sorokin, "Light Characteristics of High-power LED Luminaire With a Cooling System Based on Heat Pipe", SPQEO, Optoelectronics and optoelectronic devices, pp. 366-371, Vol. 22, No. 3, 2019. https://doi.org/10.15407/ spqeo22.03.366

[4] R-T. Wang, J-C. Wang, "Analyzing the Structural Designs and Thermal Performance of Nonmetal Lighting Devices of LED Bulbs", International Journal of Heat and Mass Transfer, pp. 750-761, Vol. 99, 2016. https://doi.org/10.1016/j.ijheatmasstransfer.2016.03.112

[5] S. Feng, Z. Liu, B. Cheng, S. Sun, T. J. Lu, F. Xu, "Design of a Novel LED Bulb With Entire Surface Thermally Activated for Passive Cooling", Applied Thermal Engineering, p. 9 pages, Vol. 198, No. 5, Nov. 2021. https://doi.org/10.1016/j.applthermaleng.2021.117466 
[6] J. Petroski, "Advanced Natural Convection Cooling Designs for Light-emitting Diode Bulb Systems, Journal of Electronic Packaging”, p. 8, Vol. 136, No. 4, 2014, 041005. https://doi. org $/ 10.1115 / 1.4028331$

[7] L. S. Leng, V. Retnasamy, M. M. Shahimin, Z. Sauli, S. Taniselass, M. H. B. A. Aziz, R. Vairavan, S. Kirtsaeng "A Comparative Study of Thermal Performance on Commercialized LED Bulb on the Luminaire Geometry", "Proc. SPIE 10124, Light-Emitting Diodes: Materials, Devices, and Applications for Solid State Lighting XXI, 2017. https://doi. org/10.1117/12.2257227

[8] E. Deng, Y. Shen, Z. Zhao, J. Li, Y. Huang, "The Algorithm and Software Implementation of the Thermal Transient Testing Technology Applied in High-Power Electronics", Sensors \& Transducers, pp. 60-66, Vol. 227, Issue 11, November 2018.

[9] V. Szekely, S. Torok, E. Nikodemusz, G. Farkas, M. Rencz, "Measurement and evaluation of thermal transients", IEEE Instrumentation and Measurement Technology Conference Budapest, Hungary, pp. 210-215. May 21-23, 2001. https://doi.org/10.1109/ IMTC.2001.928814

[10] S. S. Alrwashdeh, "Modelling of Operating Conditions of Conduction Heat Transfer Mode Using Energy 2D Simulation", iJOE International Journal of Online and Biomedical Engineering, pp. 200-207, Vol. 14, No. 9, 2018. https://doi.org/10.3991/ijoe.v14i09.9116

[11] L. Mitterhuber, "Structure Function Based Evaluation of the Thermal Behavior of an LED", master's thesis, TU Graz, 99 pages, 2015.

[12] S. Fukunaga, T. Funaki, "Transient Thermal Network Model Identification for Power Module Packages", Nonlinear Theory and Its Applications, IEICE, Vol. 11, No. 2, pp. 157-169, 2020. https://doi.org/10.1587/nolta.11.157

\section{Author}

Mika Maaspuro received the M.Sc. degree in electrical engineering (electronics) from Tampere University of Technology, Finland in 1989 and the Lic.Sc. degree (electronics) from Faculty of Mathematics and Natural Sciences in Turku University, Finland in 2010. He currently has a student position in the department of electrical engineering and automation in Aalto University, Finland.

Article submitted 2021-10-28. Resubmitted 2021-11-29. Final acceptance 2021-12-01. Final version published as submitted by the authors. 